\title{
Abacavir hypersensitivity: a model system for pharmacogenetic test adoption
}

\author{
Myla Lai-Goldman, MD, and Hawazin Faruki, DrPh
}

\begin{abstract}
A pharmacogenetic marker for abacavir hypersensitivity is rapidly being incorporated into routine medical practice following demonstration of strong clinical utility in pivotal clinical studies. As one of the few pharmacogenetic markers that have crossed from research tools to clinical adoption and utilization, the abacavir hypersensitivity pharmacogenetic marker provides a great model for demonstration of factors that are critical to successful pharmacogenetic test adoption. Several examples of novel diagnostic test implementation are reviewed with focus on factors that are critical to translation into clinical practice. Other pharmacogenetic markers that have not yet been integrated into routine clinical care are discussed and reasons for their lack of acceptance are suggested.
\end{abstract}

Genet Med 2008:10(12):874-878.

In July 2007, at the International Aids Society Conference in Sydney, Australia, data from two critical studies evaluating an abacavir hypersensitivity pharmacogenetic (PGx) marker were presented. Results from a multicenter prospective global study and a retrospective case-control study definitively validated use of $H L A-B^{\star} 5701$ screening to avoid a potentially fatal druginduced hypersensitivity reaction. ${ }^{1,2}$ Within weeks of those presentations, testing volumes for $H L A-B^{\star} 5701$ at a US-based national reference laboratory were on the rise, increasing 5 -fold over a 6-month period of time. During a time when much is discussed about the potential of personalized medicine, we are witnesses to a current example of translational medicine in practice. What created the environment in the United States to allow clinical practice to change so quickly for this pharmacogenetic marker? This paper will detail the factors leading to adoption of $H L A-B^{\star} 5701$ screening in the United States and explore opportunities to learn from this example as we anticipate the introduction of other PGx markers supporting personalized medicine.

Abacavir, an effective drug used for the management of human immunodeficiency virus (HIV)-infected patients, was granted marketing approval in the United States in 1998. Although abacavir is generally well tolerated, it has been found to cause a drug hypersensitivity reaction in $5-8 \%$ of patients initiating therapy. In 2001, several years after the drug had been

\footnotetext{
From the National Office of Science \& Quality, Laboratory Corporation of America, Burlington, North Carolina.

Hawazin Faruki, Drph, 128 East Maple Avenue, 2nd Floor, Burlington, NC 27215. E-mail: farukih@labcorp.com.

Both authors contributed equally to this paper.

Disclosure: Myla Lai-Goldman and Hawazin Faruki are both full-time employees of Laboratory Corporation of America, a for profit national clinical reference laboratory where the HLA-B*5701 test for abacavir hypersensitivity was introduced and continues to be performed. Submitted for publication August 14, 2008.

Accepted for publication September 15, 2008.
}

DOI: 10.1097/GIM.0b013e31818de71c on the market, the association of $H L A-B^{\star} 5701$ with abacavir hypersensitivity in white HIV-infected patients was first identified. ${ }^{3,4}$ From 2001 to 2006, there was much controversy about the utility of $H L A-B^{\star} 5701$ in various ethnic and geographic populations, about the potential impact that a seemingly imperfect screen would have on clinical vigilance for hypersensitivity reactions, and on whether the HLA-B $B^{\star} 5701$ screen for abacavir hypersensitivity should or should not be recommended. ${ }^{5,6}$ Lack of well-established clinical utility resulted in minimal utilization of the test in the United States throughout that period from 2001 to 2006. For a detailed review of the history of the HLA-B ${ }^{\star} 5701$ marker from discovery to the present, the reader is referred to the recent publication by Hughes et al. ${ }^{7}$

Despite the ongoing controversy regarding clinical utility of the HLA-B ${ }^{\star} 5701$ PGx marker, a cost-effectiveness study was published in 2004 by a group in the United Kingdom indicating that the test was cost-effective as a screen before administering abacavir. ${ }^{8}$ This led to increased awareness of the PGx marker, and in late 2004 our laboratory introduced HLA$B^{\star} 5701$ testing as a clinical offering for increased risk of abacavir hypersensitivity. The introduction of the test followed recognition that a small number of United States HIV-treating physicians were already requesting generic HLA testing for this purpose. Volumes were minimal, but because of ongoing concerns about racial differences and residual risk following negative results, test reports were redesigned to include racially specific $H L A-B^{\star} 5701$ prevalence rates and a formal post-test communication process was implemented. ${ }^{9}$ Test utilization remained low and ordering was primarily confined to a small group of physicians.

The status quo shifted dramatically in 2007. Aided by skin patch testing, which could be used to confirm a suspected abacavir hypersensitivity reaction, several critical studies were completed in 2007 that demonstrated generalizability of the $H L A-B^{\star} 5701$ test across racial and geographic populations and that demonstrated clinical utility with a near-perfect negative predictive value. ${ }^{1,2}$ Studies showed that screening for HLA- 
$B^{\star} 5701$, with avoidance of abacavir in those that are positive, resulted in elimination of confirmed hypersensitivity reactions to abacavir. Earlier studies showing variable performance in different racial groups were confounded by the inclusion of phenotypically heterogeneous cases. Symptoms of abacavir hypersensitivity are often nonspecific and can imitate other conditions commonly seen in HIV patients on antiviral therapy; thus, the diagnosis can be quite challenging. The use of skin patch testing allowed confirmation of clinically suspected abacavir hypersensitivity reactions and an accurate assessment of the performance characteristics of the test. Within weeks of presentation of the data from two critical drug manufacturersponsored studies, ${ }^{1,2}$ requests for the test started increasing as HIV practitioners adopted HLA-B ${ }^{\star} 5701$ screening into their standard patient care. The test reports were modified to reflect the emerging data and we discontinued the postresult follow-up call because it was no longer necessary.

Our laboratory has been a participant in introduction of many new tests and has witnessed numerous instances of transition of novel testing from low volume, primarily research and very specialized applications, to full clinical adoption and incorporation into the standard of care. On the basis of our experience, we have modeled the life cycle of new clinical laboratory tests as shown in Figure 1. The early relatively stable part of the curve may go on for years, mediated by a few early adopters who use the test in specialized situations. This low volume/ limited physician ordering pattern typically continues until a pivotal event(s) occurs establishing clinical utility, which leads to incorporation of the test into practice guidelines and/or consensus statements and the associated rapid uptake of test- ing. Eventually a plateau is reached. In the case of $H L A-B{ }^{\star} 5701$, the early portion of the curve not only included tests ordered as indicated for pre-abacavir therapy screening, but also included tests ordered for a more specialized situation - to assist in clarifying a previous suspicious history of abacavir hypersensitivity. ${ }^{9}$ Establishment of clinical utility, which occurred in 2007, resulted in logarithmic increase in test ordering as demonstrated in Figure 2 showing actual $H L A-B{ }^{\star} 5701$ test orders received.

Pivotal events that drive transition into clinical practice are usually large well-designed and controlled prospective trials, conducted under the direction of well-respected investigators, which demonstrate clinical utility of the test in a definitive manner. Publication of definitive indications for testing usually lead, shortly thereafter, to inclusion of testing in practice guidelines and/or test endorsements in consensus statements. Several examples of this scenario come to mind from our recent experience. HIV resistance testing was introduced at LabCorp in 1997 with minimal clinical acceptance until publication of two prospective trials, Viradapt and GART, in 1999 and 2000 , respectively. ${ }^{10,11}$ The two studies showed improved outcomes for patients whose choice of drug cocktail was guided by the use of resistance testing. The publication of clinical utility data, followed by inclusion of HIV resistance testing in the Department of Health and Human Services (DHHS) guideline $^{12}$ and the International Aids Society-USA treatment guideline ${ }^{13}$ had a profound impact on utilization of the test. The same pattern was seen for human papilloma virus molecular testing, which was available at our laboratory since 1988, but experienced rapid increase in volume following publica-

\section{New Test Adoption Curve}

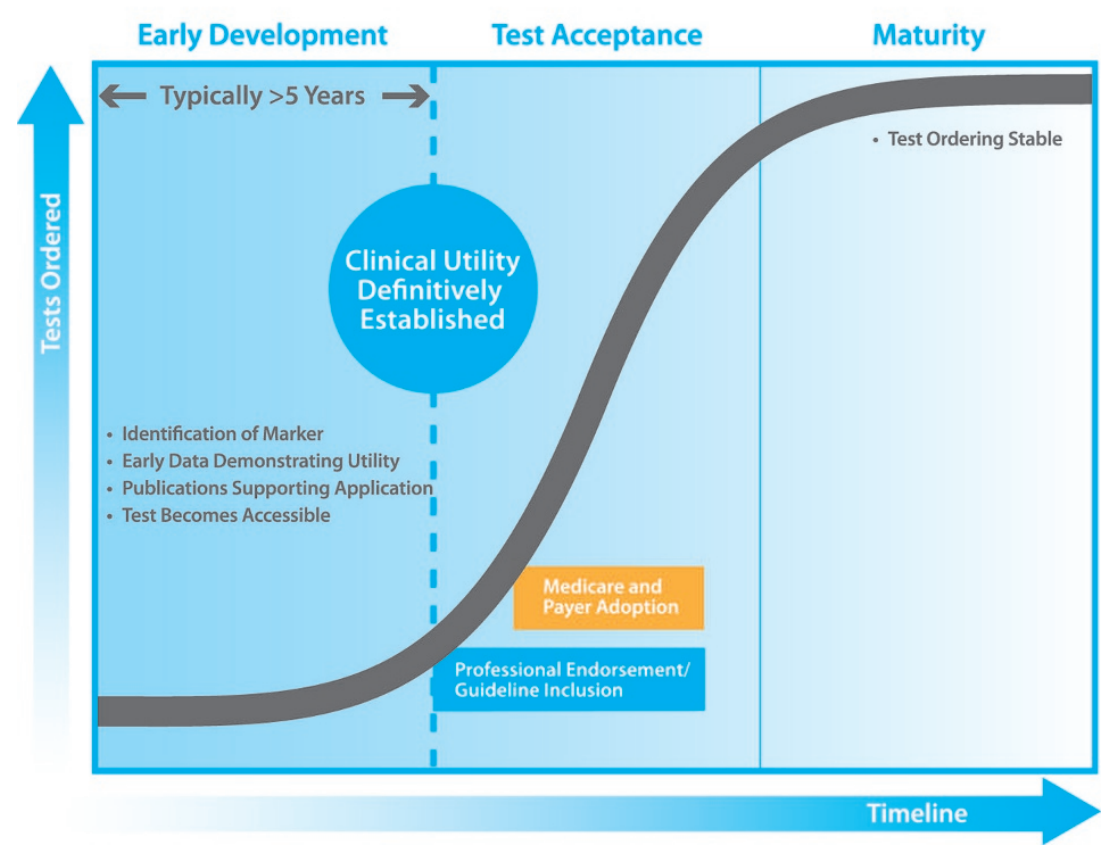

Fig. 1. New test adoption curve. 
HLA-B*5701 test orders by Qr 2002-2008

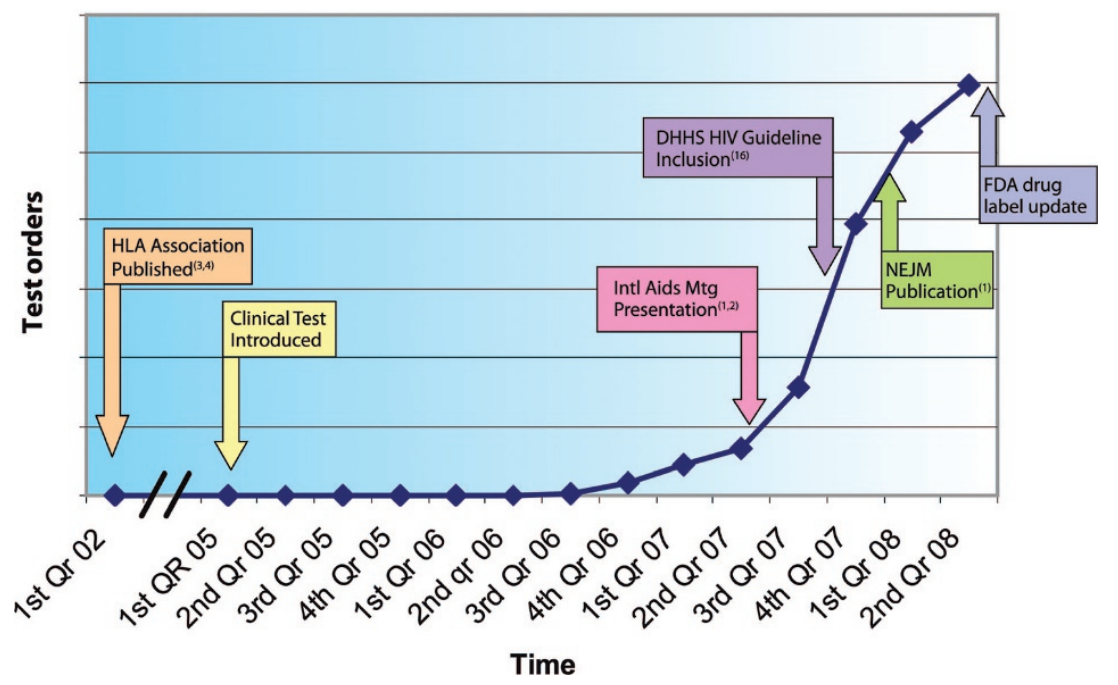

Fig. 2. Actual $H L A-B^{*} 5701$ test orders by quarter.

tion of the ASCUS/LSIL Triage Study (ALTS) study in 2001 documenting clinical utility ${ }^{14}$ and the subsequent update of the consensus guidelines for cervical cytology abnormalities. ${ }^{15}$

The HLA-B ${ }^{\star} 5701$ PGx test, like the examples reviewed above, had been available through HLA testing labs for several years with minimal acceptance into routine practice despite publication of the original association with abacavir hypersensitivity. Following pivotal studies documenting clinical utility and incorporation of testing into the DHHS guidelines of $2007,{ }^{16}$ an associated rapid uptake into clinical practice took place.

Coding and reimbursement have been identified as limiting factors in test adoption. Existing coding and reimbursement systems for clinical laboratory diagnostics generally tie reimbursement for new technologies and procedures to reimbursement for existing technologies deemed comparable, often resulting in undervaluation. The current procedural terminology (CPT) code system ${ }^{17}$ used for clinical laboratory diagnostics is a cost- and procedure-based, rather than valuebased, system, and often fails to account for all costs and procedures associated with a given assay. Assay development costs and intellectual property licensing costs (e.g., royalties) are not covered by the CPT system, even though they may represent the most significant costs to a laboratory providing a novel test. Additionally, there may be procedures, and therefore costs incurred, with no applicable code. When there is no CPT code for a particular procedure or test, the process of securing a code and fair payment policy for that new code can take several years. Therefore, depending on the methodology used, current CPT coding may or may not provide opportunity for adequate reimbursement for testing performed. When applicable CPT codes are available, and clinical utility has been established, adequate reimbursement may still be challenged by individual pay or policies. Fortunately, for the PGx marker $H L A-B^{\star} 5701$, the standard molecular procedural codes were appropriate and had been used for years to code HLA testing; thus, reimbursement was not a significant barrier.

Cost-effectiveness may also play a role in the transition from limited utilization to full adoption into clinical practice; however, it is not a very effective driver on its own. A cost-effectiveness study of the $H L A-B^{\star} 5701$ test published in $2004^{8}$ seems to have had limited influence on the adoption of the test. It was not until clinical utility was established that cost-effectiveness became a facilitator of test adoption. An additional cost-effectiveness study was recently presented, ${ }^{18}$ thereby strengthening the case for third party reimbursement. The lack of cost-effectiveness data, however, is not sufficient to deter utilization. There are numerous examples of clinical tests in routine practice for which no costeffectiveness studies exist.

Other factors such as FDA clearance of in vitro diagnostic kits for clinical assays and/or inclusion by the FDA of a test in the labeling of a drug have traditionally been thought of as significant contributors to acceptance of clinical laboratory tests into general practice. Our recent experience with several PGx markers would suggest otherwise. Both UGT1A1 and CYP450 (more specifically the CYP2C19 and CYP2D6 combination test) have FDA-cleared assays that have been available at our laboratory since their approval in 2005; however, to date, there has been minimal adoption of either test into clinical practice. The $U G T 1 A 1^{\star} 28$ marker for predicting toxicity to the chemotherapeutic drug irinotecan was added to the drug labeling in 2005, but is not required for drug administration (package insert). Geographic and ethnic differences in test performance prevail, and drug dosing seems to alter the usefulness of the test. ${ }^{19,20}$ For CYP450 testing, despite significant interest from the pharmaceutical industry, there has been very limited community practice utilization. Unlike $H L A-B^{\star} 5701$, there is a lack of strong and definitive prospective trial data supporting clinical utility of either UGT1A1 for irinotecan toxicity or CYP450 drug metabolism testing. One application of CYP450 
testing, that had been initially promoted to assist in selective serotonin reuptake inhibitors antidepressant therapy management, has now been specifically discouraged because of lack of sufficient data demonstrating clinical utility. ${ }^{21}$

The FDA's inclusion of references to genetic testing (CYP2C9 and VKORC1) in the drug labeling of warfarin in 2007 has, likewise, not resulted in significant test adoption. Concerns about clinical utility, how to use the genetic information in dosing warfarin, and lack of prospective data demonstrating an improved outcome has delayed translation into everyday practice. Indeed, a small recent prospective study, the Couma-Gen study, on the use of warfarin PGx testing in determining dosing failed to demonstrate a reduction in adverse events. ${ }^{22}$ The study demonstrated the potential for PGx testing to reduce the number of dose adjustments needed and the number of international normalized ratio measurements needed to reach a stable dose, but did not show a significant improvement in clinical outcomes. ${ }^{22}$ The choice of control group for the study may be critical. Although PGx testing may only have minimal advantage for the highly expert thrombosis practitioners, it may provide benefit for less-specialized physicians prescribing warfarin. Recent education efforts from the Critical Path Institute and the American Medical Association ${ }^{23}$ are helpful, but the pivotal studies demonstrating clinical utility are needed first to impact widespread adoption. To date, there are no FDA-cleared kits for $H L A-B^{\star} 5701$ testing. Although the FDA recently (July 2008) included HLA-B ${ }^{\star} 5701$ testing in the drug label of abacavir, it was not the critical event impacting widespread acceptance (see Fig. 2). Demonstration of strong clinical utility was sufficient to drive widespread adoption.

In addition to favorable data supporting clinical utility, other factors unique to HIV practitioners using the test have impacted test adoption in the HIV-treating community. The HIV-treating physician group tends to be a relatively small and closely knit group that is accustomed to sharing and incorporating new HIV developments into their patient management. HIV practitioners are well versed in using molecular testing to select and adjust drug regimens because HIV-1 viral load and resistance testing have been a routine part of HIV patient care for many years. HLA- $B^{\star} 5701$ molecular testing to assist in selecting an antiretroviral regimen, therefore, did not pose a new barrier. The ever-changing landscape of anti-HIV therapeutics had already created an environment where expeditious incorporation of the latest scientific advances into the care and management of HIV patients was customary. Consequently, there has been rapid adoption of $H L A-B{ }^{\star} 5701$ testing among HIV practitioners. Adoption of PGx markers by other groups of practitioners may not be as efficient.

Finally, access to the test is a necessary feature of widespread utilization. Limited access can slow adoption even when clinical utility has been clearly established. In a recent publication regarding integration of a new PGx test requiring antiretroviral drug into clinical practice in Europe, Vandekerckhove et al. ${ }^{24}$ suggest that wide use of the HIV drug maraviroc may be "currently hampered by the lack of a readily available R5virus only determination test (tropism test)." The viral tropism test is a necessary step to determine drug eligibility, but to date only one test offered by a single laboratory has been clinically validated. Specimen transport complications, turn around time, expense, etc. associated with single source availability have resulted in limited access globally. ${ }^{24}$

An alignment of a variety of factors has led to rapid adoption of the HLA-B ${ }^{\star} 5701$ test. The well-controlled prospective study and the $H L A-B^{\star} 5701$ test negative predictive value were most influential. Adoption was further facilitated by having adequate coding and reimbursement in place, by data supporting cost-effectiveness, by involvement of a progressive HIVtreating physician group, and by widespread access to the test. Numerous associations of genetic markers with drug toxicities have been observed but very few have been incorporated into routine health care. Adoption of PGx tests into routine health care will take investment in prospective, well-controlled studies to demonstrate clinical utility. The pharmaceutical industry has historically been reluctant to support PGx screening in association with administration of their drugs. This may now be changing as exemplified by the GlaxoSmithKline sponsorship of studies of the PGx marker, HLA-B ${ }^{\star} 5701$. Studies have shown that when $H L A-B^{\star} 5701$ screening is used, physicians aware of the $H L A-B^{\star} 5701$ negative result are less likely to diagnose an inaccurate clinical abacavir hypersensitivity. $1,25,26$ This allows a greater number of patients to continue abacavir therapy. Consequently, it seems that $H L A-B^{\star} 5701$ screening may in fact increase the use of the drug, because many nonsubstantiated clinical diagnoses of abacavir hypersensitivity can be avoided when screening is implemented.

The success of $H L A-B^{\star} 5701$ as a PGx screening test, coupled with the increased awareness of the impact of drug toxicity reactions and greater interest in personalized medicine, should encourage all stakeholders to support adequately powered and controlled prospective clinical validation studies. Much unrealized potential to improve health outcomes is waiting for pivotal studies of PGx markers demonstrating clinical utility. Our experience with $H L A-B^{\star} 5701$ is a great example of what pivotal studies demonstrating clinical utility can accomplish.

\section{References}

1. Mallal S, Phillips E, Carosi G, et al. HLA-B ${ }^{\star} 5701$ screening for hypersensitivity to abacavir. N Engl J Med 2008;358:568-579.

2. Saag M, Balu R, Brachman P, et al. High sensitivity of $H L A-B^{\star} 5701$ in Whites and Blacks in immunologically-confirmed cases of hypersensitivity (ABC HSR). Clin Infect Dis 2008;46:1111-1118.

3. Mallal S, Nolan D, Witt C, et al. Association between the presence of HLA-B ${ }^{\star} 5701$, HLA-DR7, and HLA-DQ3 and hypersensitivity to the HIV-1 reverse-transcriptase inhibitor abacavir. Lancet 2002;359:727-732.

4. Heatherington S, Hughes AR, Mosteller M, et al. Genetic variations in HLA-B region and hypersensitivity reactions to abacavir. Lancet 2002;359:1121-1122.

5. Martin AM, Nolan D, Gaudieri S, et al. Predisposition to abacavir hypersensitivity conferred by HLA-B ${ }^{\star} 5701$ and a haplotypic Hsp70-Hom variant. Proc Natl Acad Sci USA 2004;14:335-342.

6. Hughes AR, Mosteller M, Bansal AT, et al. Association of genetic variations in HLA-B region with hypersensitivity to abacavir in some, but not all, populations. Pharmacogenomics 2004;5:203-211.

7. Hughes AR, Spreen WR, Mosteller M, et al. Pharmacogenetics of hypersensitivity to abacavir: from PGx hypothesis to confirmation to clinical utility. Pharmacogenomics J 2008;8:365-374. 


\section{Lai-Goldman and Faruki}

8. Hughes DA, Vilar FJ, Ward CC, Alfirevic A, Park BK, Pirmohamed M. Cost-effectiveness analysis of $\mathrm{HLA} \mathrm{B}^{\star} 5701$ genotyping in preventing abacavir hypersensitivity. Pharmacogenetics 2004;14:1-8.

9. Faruki H, Heine U, Brown T, Koester R, Lai-Goldman M. HLA-B ${ }^{\star 5701 ~ c l i n i c a l ~}$ testing: early experience in the United States. Pharmacogenet Genomics 2007;17: 857-860.

10. Durant J, Clevenbergh P, Halfon P, et al. Drug-resistance genotyping in HIV-1 therapy: the VIRADAPT randomised controlled trial. Lancet 1999;353:2195-2199.

11. Baxter JD, Mayers DL, Wentworth DN, et al. A randomized study of antiretroviral management based on plasma genotypic antiretroviral resistance testing in-patients failing therapy. CPCRA 046 Study Team for the Terry Beirn Community Programs for Clinical Research on AIDS. AIDS 2000;14:F83-F93.

12. US Department of Health and Human Services and the Henry J Kaiser Family Foundation. Guidelines for the use of antiretroviral agents in HIV-infected adults and adolescents. Available at: http://www.hivatis.org/trtgdlns.html. Accessed January $28,2000$.

13. Hirsch M, Brun-Vezinet F, D’Aquila R, et al. Antiretroviral drug resistance testing in adult HIV-1 infection: recommendations of an International AIDS Society-USA Panel. JAMA 2000;283:2417-2426.

14. Solomon D, Schiffman M, Tarone R; ALTS Study Group. Comparison of three management strategies for patients with atypical squamous cells of undetermined significance: baseline results from a randomized trial. J Natl Cancer Inst 2001;93: 293-299.

15. Wright TC, Coy JT, Massad LS, Twiggs LB, Wilkinson EJ. ASCCP-sponsored consensus conference. 2001 consensus guidelines for the management of women with cervical cytological abnormalities. JAMA 2002;287:2120-2129.

16. US Department of Health and Human Services Guidelines for the use of antiretroviral agents in HIV-infected adults and adolescents. Available at: http://AIDSinfo.nih.gov. Accessed December 1, 2007.

17. American Medical Association. Current Procedural Terminology Coding. Available at: http://www.ama-assn.org/ama/pub/category/3113.html. Accessed July 2008.
18. Schackman B, Scott C, Walensky R, Losina E, Freedberg KA, Sax PE. The cost effectiveness of HLA-B ${ }^{\star} 5701$ genetic screening to guide initial antiretroviral therapy for HIV. AIDS 2008;22:2025-2033.

19. Hoskins JM, Goldberg RM, Qu P, Ibrahim JG, McLeod HL. UGT1A1²8 genotype and irinotecan-induced neutropenia: dose matters. J Natl Cancer Inst 2007;99: 1290-1295.

20. Han JY, Lim HS, Shiun ES, et al. Comprehensive analysis of UGT1A polymorphisms predictive for pharmacokinetics and treatment outcome in patients with non-smallcell lung cancer treated with irinotecan and cisplatin. J Clin Oncol 2006;24:22372244 .

21. Evaluation of Genomic Applications in Practice and Prevention (EGAPP) Working Group. Recommendations from the EGAPP Working Group: testing for cytochrome P450 polymorphisms in adults with nonpsychotic depression treated with selective serotonin reuptake inhibitors. Genet Med 2007;9:819-825.

22. Anderson JL, Horne BD, Stevens SM, et al. Randomized trial of genotype-guided versus standard warfarin dosing in patients initiating oral anticoagulation. Circulation 2007;116:2563-2570.

23. American Medical Association, Arizona CERT, \& Critical Path Institute Personalized Health Care Report 2008: Warfarin and Genetic Testing. Available at: http:// www.ama-assn.org/ama1/pub/upload/mm/464/warfarin_brochure.pdf. Accessed March 2008.

24. Vandekerckhove L, Verhofstede C, Vogelaers D. Maraviroc: integration of a new antiretroviral drug class into clinical practice. J Antimicrob Chemotherapy 2008;61: 1187-1190.

25. Rauch A, Nolan D, Martin A, McKinnon E, Almeida C, Mallal S. Prospective genetic screening decreases the incidence of abacavir hypersensitivity reaction in the western Australian HIV cohort. Clin Infect Dis 2006;43:99-102.

26. Zucman D, de Truchis P, Majerholc C, Stegman S, Caillat-Zucman S. Prospective screening for human leukocyte antigen- $B^{\star} 5701$ avoids abacavir hypersensitivity reaction in the ethnically mixed French HIV population. J Acquir Immune Defic Syndr 2007;45:1-3. 